\title{
PopPAnTe: population and pedigree association testing for quantitative data
}

\author{
Alessia Visconti ${ }^{*}$ (D), Mashael Al-Shafai2,3,4,5, Wadha A. Al Muftah2,3,4, Shaza B. Zaghlool3, \\ Massimo Mangino ${ }^{1,6}$, Karsten Suhre $^{3 \dagger}$ and Mario Falchi ${ }^{1 \dagger}$
}

\begin{abstract}
Background: Family-based designs, from twin studies to isolated populations with their complex genealogical data, are a valuable resource for genetic studies of heritable molecular biomarkers. Existing software for family-based studies have mainly focused on facilitating association between response phenotypes and genetic markers, and no user-friendly tools are at present available to straightforwardly extend association studies in related samples to large datasets of generic quantitative data, as those generated by current -omics technologies.

Results: We developed PopPAnTe, a user-friendly Java program, which evaluates the association of quantitative data in related samples. Additionally, PopPAnTe implements data pre and post processing, region based testing, and empirical assessment of associations.

Conclusions: PopPAnTe is an integrated and flexible framework for pairwise association testing in related samples with a large number of predictors and response variables. It works either with family data of any size and complexity, or, when the genealogical information is unknown, it uses genetic similarity information between individuals as those inferred from genome-wide genetic data. It can therefore be particularly useful in facilitating usage of biobank data collections from population isolates when extensive genealogical information is missing.
\end{abstract}

Keywords: Association studies, Heritability, -omics data, Family data, Isolated population, Population genetics

\section{Background}

Family-based designs, from complex genealogical structure to twin studies, are a valuable resource for genetic studies. The primary aim of currently-available software accounting for population substructure and/or relatedness in the statistical model (e.g., EMMA [1], Merlin [2], GenABEL [3], QTDT [4]) is to evaluate the association between genetic SNP markers and response phenotypes and, to date, very few tools are available to test the association of large quantitative datasets generated by highthroughput -omics technologies (e.g., epigenomic versus metabolomic data, or transcriptomic versus metagenomic data) in familial samples. For instance, although modelling of genealogical data can be performed by the coxme [5]

\footnotetext{
*Correspondence: alessia.visconti@kcl.ac.uk

${ }^{\dagger}$ Equal contributors

'Department of Twin Research and Genetic Epidemiology, King's College London, London, UK

Full list of author information is available at the end of the article
}

and kinship2 [6] $\mathrm{R}$ packages, $\mathrm{R}$ is not a particularly efficient environment to carry out hundred of thousands or millions tests.

We have implemented a user-friendly Java program, PopPAnTe, to perform exact association tests between large quantitative datasets in family-based studies. Relationships between individuals can be described either by known pedigrees of any size and complexity or by genetic similarity matrices (GSMs) inferred from genome-wide genetic data [7]. Pedigree-based and pedigree-free relatedness can show some discordance, especially when some degree of hidden relatedness or population substructure is observed in the data and extensive genealogical information is missing or incomplete. For instance, genealogical information going back more than three or four generations may be difficult to be retrieved for individuals recruited in large-scale biobank started in genetic isolates such as those from the Middle East. 


\section{Implementation}

PopPAnTe assesses the relationship between quantitative dependent variables (responses) and quantitative independent variables (predictors) within a variance components framework in order to model the resemblance among relatives.

The association of a single predictor with a single response variable is described as

$$
r_{i}=\mu+\beta p_{i}+\sum_{j} \psi_{i j} C_{i j}+g_{i}+e_{i}
$$

where $r_{i}$ represents the response value for the $i$-th individual, $\mu$ the response mean, $\beta$ the estimate of the predictor value $p_{i}, \psi_{j}$ the estimate of the $j$-th covariate $C$, and $g_{i}$ and $e_{i}$ the polygenic and environmental effect, respectively.

The total response variance is partitioned into polygenic and environmental variances (the latter including also measurement errors), and the variance-covariance matrix is calculated as

$$
\omega=2 \Phi \sigma_{a}^{2}+I \sigma_{e}^{2}
$$

where $\Phi$ is the relatedness matrix between each pair of individuals, $I$ is the identity matrix, and $\sigma_{a}^{2}$ and $\sigma_{e}^{2}$ are the additive genetic and environmental variance, respectively.

Within the same framework, PopPAnTe allows the evaluation of the narrow heritability of any quantitative response variable included in the analysis.

The significance of the association is calculated using a formal likelihood-ratio test comparing the likelihood of the alternative model described in Eq. (1) to the likelihood of a null model where the effect of the predictor is constrained to zero.

PopPAnTe implements an exact linear mixed model equivalent to that implemented in the QTDT software [4].

To speed-up the evaluation, PopPAnTe clusters variables having the same pattern of missingness (i.e., the same missing values in a subset of individuals), then evaluates the likelihood of the null model once, and reuses the value to assess every variable included in the same cluster. PopPAnTe also allows the evaluation of empirical $p$ values by randomly permuting the predictor values among subjects and re-assessing the association under the null hypothesis. When genealogical information is provided as input, predictor values are randomly permuted within families in order to preserve the phenotypic correlation between family members. To speed up performance, PopPAnTe implements an adaptive permutation approach [8], stopping the generation of randomly permuted samples earlier when there is little or no evidence of significance.

\section{Pedigree versus population analysis}

When genealogical information is available, PopPAn'Te evaluates the relatedness matrix from the known pedigree relationships using a recursive procedure and assuming pedigree founders as unrelated [9]. This results in a variance-covariance matrix that is usually both symmetric and semi-positive definite. Therefore, the maximum likelihood estimates of the variance components can be assessed through efficient Cholesky decomposition.

When the genealogical information is not available, a GSM can be estimated from genome-wide genetic data with any of several well-established tools, such as PLINK [10], GCTA [11], or LDAK [12], and given as input to PopPAnTe. The property of positive-definiteness does not always hold for GSMs. A bending procedure [13] is used by default to transform the matrix when it is not positive semi-definite -but the user has the option to use a LU decomposition instead. Additionally, PopPAnTe implements the QR decomposition to solve the rare cases where the variance-covariance matrix is not invertible and neither the Cholesky nor the LU decompositions can be used.

To speed-up the evaluation of the variance components, PopPAnTe allows the user to set an arbitrary threshold below which individuals can be considered as unrelated. Otherwise, the user has the option of using the value of expected kinship between second or third cousins $[14,15]$.

\section{Region-based testing}

When predictors can be ordered in space, as in the case of epigenetic markers, PopPAnTe allows the computation of region-based association tests by gathering information from flanking predictors included in a sliding window of user-defined size, whose values are replaced by their first principal component. By definition the first principal component accounts for as much of the variability in the data as possible, and can thus be used to summarise the joint distribution of all variables included in a given region for gene- or region-based association studies (e.g., $[16,17])$.

\section{Data pre- and post-processing}

Quantile normalisation [18] can be automatically applied to improve normality of both response variables and predictors. Moreover, PopPAnTe implements two approaches to correct the association test for unwanted biological and technical variability (e.g., batch effects). When the source of the confounders is known, it can be directly included in the association model. To deal with unknown sources of biological and technical co-variation, PopPAnTe can integrate into the association model the principal components that are required to explain a user-specified percentage of variation.

PopPAnTe implements the Benjamini-Hochberg procedure (BH step-up procedure) to control the false discovery 
rate [19], and, to aid in results interpretation and further analyses, it generates basic Quantile-Quantile and Manhattan plots - the latter only when genomic data that can be ordered in space (e.g., CpG loci) are used as predictors.

Finally, when the genealogical information is available, to determine whether an association has been generated by a uniform contribution of all the families within the sample, or by a strong contribution of a small number of families, PopPAnTe reports, for each test, the percentage of families showing a positive contribution and the Gini coefficient [20] assessed on family contribution to the $\chi^{2}$ statistics.

\section{Results and discussion}

We carried out a simulation study to estimate PopPAnTe's computational requirements. We also presented two realworld case studies (an outbred and an inbred sample), showing the results obtained through both pedigreebased kinship matrix and GSM (evaluated using different software).

\section{Simulation study}

We simulated quantitative response and predictor variables in three-generation families (maternal and paternal grandparents, parents, and two offspring).

In the first simulated scenario, we aimed to test the relationship between running time and number of subjects included in the analysis. Therefore, we simulated 11 independent datasets including an increasing number of three-generation families (from 10 to 1000, thus comprising 80 to 8000 individuals) and one response and one predictor variable for all simulated subjects.

In the second simulated scenario, we aimed to test the relationship between running time and number of variables included in the analysis. Consequently, we fixed the number of families included in each dataset (125 families, corresponding to 1000 individuals) and generated 7 independent datasets with one response and an increasing number of predictors ranging from one to 10,000 .

Both scenarios were simulated 100 times and the median time necessary for the testing step recorded. Simulations were performed on a Mac BookPro $2.3 \mathrm{GHz}$, Intel Core i7, 16 GB RAM; Java version 1.7. Default parameters were used for the Java Virtual Machine (1 GB of memory, 1 thread).

Tables 1 and 2 show the median running time for each simulated scenario. We observed a linear relationship between running time and both samples size and number of tests. As expected, when multiple tests were performed (second scenario), the per-test running time decreased, due to the fact that PopPAnTe clusters variables having the same pattern of missingness and evaluates the likelihood of the null model only once.
Table 1 Results of the first simulated scenario

\begin{tabular}{lll}
\hline Family number & Population size & Time $(\mathrm{ms})$ \\
\hline 10 & 80 & 58 \\
20 & 160 & 78 \\
30 & 240 & 100 \\
40 & 320 & 132 \\
50 & 400 & 141 \\
100 & 800 & 200 \\
125 & 1000 & 240 \\
250 & 2000 & 366 \\
500 & 4000 & 692 \\
750 & 6000 & 988 \\
1000 & 8000 & 1287 \\
\hline
\end{tabular}

One response and one predictor variable were simulated for each subject. Each dataset was simulated 100 times and the median time necessary for the testing step reported

\section{Case study 1: Epigenome-wide association study in a Qatari family study}

We carried out an epigenome-wide association study of body mass index (BMI) using extended families from Qatar. The Qatari population is an isolated inbred population characterised by a large number of consanguineous families [21]. A detailed description of the subjects and methylation data included in this study has been previously reported in Zaghlool et al. [22]. Briefly, we used genome-wide methylation and SNP data generated from whole blood on the Infinium HumanMethylation450 Bead-Chip (Illumina Inc, San Diego, CA) and the Illumina HumanOmni2.5-8M BeadChip, respectively. DNA methylation Beta-values were measured for 123 individuals, 88 with both genotype and BMI data in 13 multigenerational families. We used GCTA to calculate a GSM between pairs of individuals using all autosomal SNP markers with minor allele frequency $>0.01$. We compared heritability estimates of the methylation values at

Table 2 Results of the second simulated scenario

\begin{tabular}{ll}
\hline Number of predictors & Time (s) \\
\hline 1 & 0.24 \\
10 & 0.94 \\
100 & 7.74 \\
500 & 37.67 \\
1000 & 47.00 \\
2500 & 119.00 \\
5000 & 238.50 \\
10000 & 479.00
\end{tabular}

The number of families included in each dataset was fixed (125 families, corresponding to 1000 individuals). Each dataset was simulated 100 times and the median time necessary for the testing step reported 
CpG loci and their association with BMI in the Qatari family study using either the family information or the inferred GSM. Age, sex, and cell-type proportions as estimated using the Houseman method [23] were included in the model as fixed effects. We observed a very high concordance correlation coefficient [24] of the effect size estimates for the association between CpG methylation states and BMI $(r \beta=0.99$; Fig. 1, left), as well as of the CpG-specific component of genetic and environmental variances $\left(r \sigma_{a}^{2}=0.99\right.$ and $r \sigma_{e}^{2}=0.90$, respectively; Fig. 1, right).

\section{Case study 2: transcriptome-wide association study in UK twins}

In the second case study, we carried out a transcriptomewide association study with BMI in a cohort of healthy female Caucasians twins. The TwinsUK adult twin registry includes about 12,000 subjects, predominately females [25]. Genotyping of the TwinsUK dataset was performed with a combination of Illumina HumanHap300, HumanHap610Q, 1M-Duo and 1.2MDuo 1M chips and imputation was performed using the IMPUTE software package (v2), as previously described [26]. Expression profiling in subcutaneous adipose tissue was measured using Illumina Human HT-12 V3 BeadChips for 825 female individuals [27], 778 of whom had both genotype data and BMI information. We used LDAK to calculate a GSM based on allelic correlation across autosomes. Before calculation, we excluded SNPs with minor allele frequency $<0.01$. We compared effect size estimates of the gene expression profiles versus BMI, using either the family information or the inferred GSM. Age was included in the model as a fixed effect. We observed a very high concordance correlation coefficient of the effect size estimates for the association between gene expression levels and BMI $(r \beta=0.99$; Fig. 2, left), as well as of the genespecific genetic component of genetic and environmental variances $\left(r \sigma_{a}^{2}=0.99\right.$ and $r \sigma_{e}^{2}=0.99$, respectively; Fig. 2, right).

\section{Conclusions}

PopPAnTe is a user-friendly platform-independent Java program that enables pairwise association testing of large numbers of predictor and response variables in related samples. PopPAnTe uses either known pedigree structures or GSMs inferred from genome-wide genetic data, allowing the user to select the best approach according to the available data. When genome-wide genetic data are available, it may be advisable to use the GSM instead of the expected kinship matrix calculated using the genealogical information $[28,29]$. PopPAnTe can thus also facilitate the usage of biobank collections from population isolates when extensive genealogical information is missing.

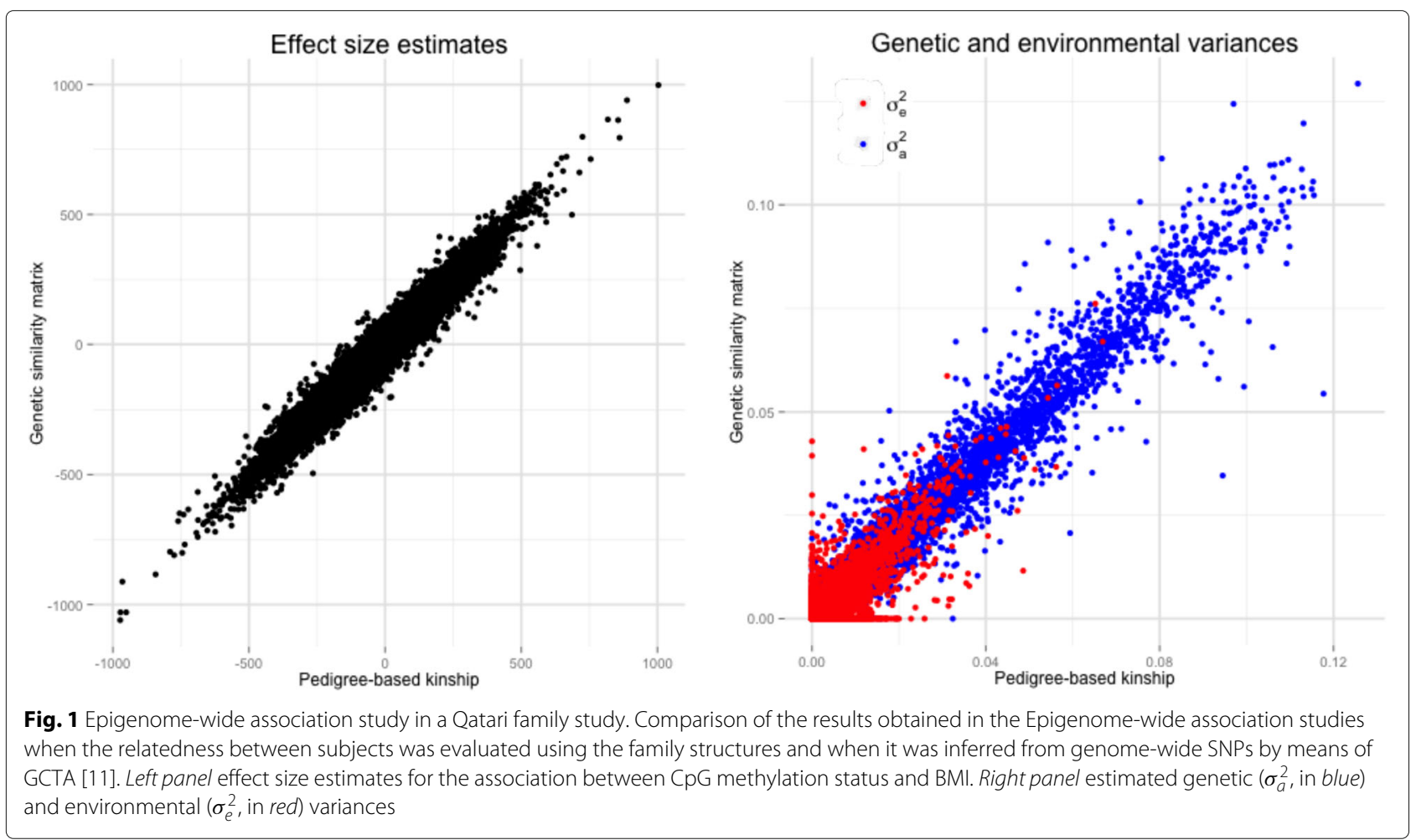




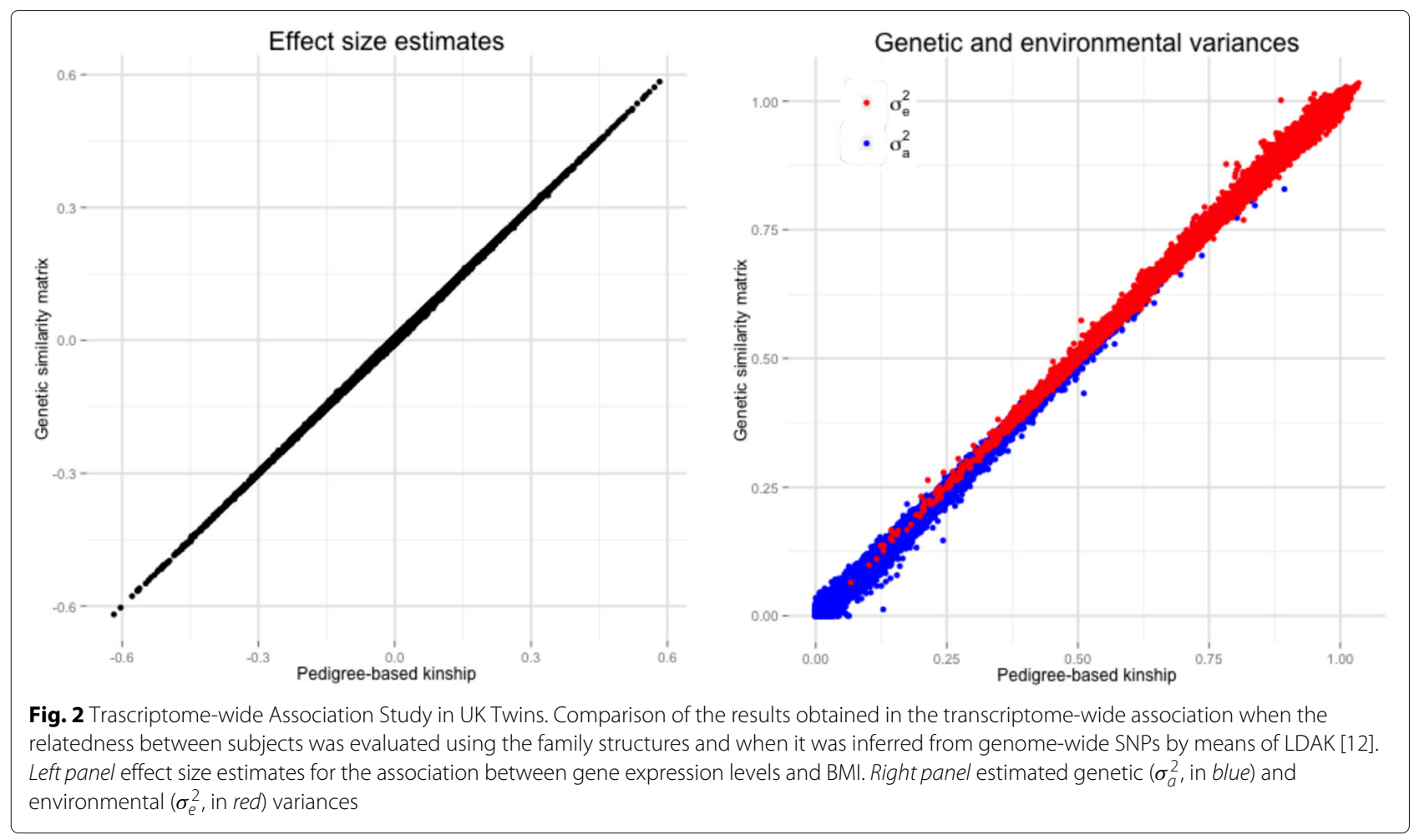

\section{Availability and requirements \\ Project name: PopPAnTe \\ Project home page: http://www.twinsuk.ac.uk/project/ poppante/ \\ Operating system(s): Platform independent \\ Programming language: Java \\ Other requirements: Java 1.7 or higher \\ License: GNU GPL 3 or higher \\ Any restrictions to use by non-academics: None}

\section{Abbreviations \\ BMI: Body mass index; GSM: Genetic similarity matrix}

\section{Acknowledgements}

We thank the Qatar Diabetes Association (QDA) and Cindy McKeon for their help in sample recruitment. AV and MF thank S. Burbidge and M. Harvey of the Imperial College High-Performance Computing service for their assistance, Doug Speed for his valuable suggestions, and Julia El-Sayed Moustafa for her constructive comments on the manuscript. We are grateful to all study participants for their contribution to this research study. The two studies were conducted in concordance with the Helsinki declaration of ethical principles for medical research involving human subjects. The studies were approved by the relevant institutional review boards in Qatar (Institutional Review Board of Weill Cornell Medical College in Qatar, ethical approval numbers 2012-003 and 2012-0025), and in the UK (Guy's and St. Thomas' Hospital Ethics Committee). Written informed consent was obtained from every participant in each study.

\section{Funding}

This work was supported by 'Biomedical Research Program' funds at Weill Cornell Medicine in Qatar, a program funded by the Qatar Foundation. MF and AV are supported by MRC grant MR/K01353X/1. TwinsUK is funded by the Wellcome Trust, Medical Research Council, European Union, the National Institute for Health Research (NIHR)-funded BioResource, Clinical Research
Facility and Biomedical Research Centre based at Guy's and St Thomas' NHS Foundation Trust in partnership with King's College London.

\section{Authors' contributions}

$A V$ and MF designed the software. AV implemented PopPAnTe, and performed the computational experiments. MAS, WAAM, SBZ, and KS generated the Qatari family study data. MM provided the data for the Twins UK cohort, and tested the software. AV and MF wrote the manuscript. All authors read, commented, and approved the final manuscript.

\section{Competing interests}

The authors declare that they have no competing interests.

\section{Author details}

${ }^{1}$ Department of Twin Research and Genetic Epidemiology, King's College London, London, UK. ${ }^{2}$ Department of Genomics of Common Diseases, Imperial College London, London, UK. ${ }^{3}$ Department of Physiology and Biophysics, Weill Cornell Medical College in Qatar, Doha, Qatar. ${ }^{4}$ Research Division, Qatar Science Leadership Program, Qatar Foundation, Doha, Qatar. ${ }^{5}$ Department of Biomedical Sciences, College of Health Sciences at Qatar University, Doha, Qatar. ${ }^{6}$ NIHR Biomedical Research Centre at Guy's and St Thomas' Foundation Trust, London, UK

Received: 30 October 2015 Accepted: 31 January 2017

Published online: 10 February 2017

\section{References}

1. Kang HM, Zaitlen NA, Wade CM, Kirby A, Heckerman D, Daly MJ, Eskin E. Efficient control of population structure in model organism association mapping. Genetics. 2008;178(3):1709-23.

2. Abecasis GR, Cherny SS, Cookson WO, Cardon LR. Merlin - rapid analysis of dense genetic maps using sparse gene flow trees. Nat Genet. 2002;30(1):97-101.

3. Aulchenko YS, Ripke S, Isaacs A, Van Duijn CM. GenABEL: an R library for genome-wide association analysis. Bioinformatics. 2007;23(10):1294-96.

4. Abecasis $\mathrm{G}$, Cardon $\mathrm{L}$, Cookson W. A general test of association for quantitative traits in nuclear families. Am J Hum Genet. 2000;66(1):279-92. 
5. Therneau T. coxme: Mixed effects Cox models. R package version 2.2.5.

6. Therneau T, Atkinson E, Sinnwell J, Matsumoto M, Schaid D, McDonnell S. kinship2: Pedigree Functions, R package version 2.1.6.4.

7. Hill WG. Understanding and using quantitative genetic variation. Philos Trans R Soc Lond B Biol Sci. 2010;365(1537):73-85.

8. Che R, Jack JR, Motsinger-Reif AA, Brown CC. An adaptive permutation approach for genome-wide association study: evaluation and recommendations for use. BioData Min. 2014;7(1):9-22.

9. Thompson EA. Pedigree Analysis in Human Genetics. Baltimore: Johns Hopkins University Press; 1986.

10. Purcell S, Neale B, Todd-Brown K, Thomas L, Ferreira MA, Bender D, Maller J, Sklar P, De Bakker PI, Daly MJ, et al. PLINK: a tool set for whole-genome association and population-based linkage analyses. Am J Hum Genet. 2007;81(3):559-75.

11. Yang J, Lee SH, Goddard ME, Visscher PM. GCTA: a tool for genome-wide complex trait analysis. Am J Hum Genet. 2011;88(1):76-82.

12. Speed D, Hemani G, Johnson MR, Balding DJ. Improved heritability estimation from genome-wide SNPs. Am J Hum Genet. 2012;91(6): $1011-21$.

13. Hayes J, Hill W. Modification of estimates of parameters in the construction of genetic selection indices ('bending'). Biometrics. 1981483-493.

14. Yang J, Benyamin B, McEvoy BP, Gordon S, Henders AK, Nyholt DR, Madden PA, Heath AC, Martin NG, Montgomery GW, et al. Common snps explain a large proportion of the heritability for human height. Nat Genet. 2010;42(7):565-9.

15. Kopps AM, Kang J, Sherwin WB, Palsbøll PJ. How well do molecular and pedigree relatedness correspond, in populations with variable mating systems, and types and quantities of molecular and demographic data? G3: Genes| Genomes| Genetics. 2014;5(9):1815-26.

16. Gauderman WJ, Murcray C, Gilliland F, Conti DV. Testing association between disease and multiple SNPs in a candidate gene. Genet Epidemiol. 2007;31(5):383-95.

17. Agerbo E, Mortensen PB, Wiuf C, Pedersen MS, McGrath J, Hollegaard MV, Nørgaard-Pedersen B, Hougaard DM, Mors O, Pedersen CB. Modelling the contribution of family history and variation in single nucleotide polymorphisms to risk of schizophrenia: a Danish national birth cohort-based study. Schizophr Res. 2012;134(2):246-52.

18. Wichura MJ. Algorithm AS 241: The percentage points of the normal distribution. Journal of the Royal Statistical Society. Series C (Applied Statistics). 1988;37(3):477-84.

19. Benjamini $Y$, Hochberg Y. Controlling the false discovery rate: a practical and powerful approach to multiple testing. J Royal Stati Soc Series B Method. 1995289-300.

20. Gini C. Reprinted in Memorie di metodologica statistica, vol. 1. 1912

21. Bener A, Alali KA. Consanguineous marriage in a newly developed country: the qatari population. J Biosoc Sci. 2006;38(02):239-46.

22. Zaghlool SB, Al-Shafai M, Al Muftah WA, Kumar P, Falchi M, Suhre K. Association of DNA methylation with age, gender, and smoking in an Arab population. Clin Epigenetics. 2015;7(1):6.

23. Houseman EA, Accomando WP, Koestler DC, Christensen BC, Marsit CJ, Nelson $\mathrm{HH}$, Wiencke JK, Kelsey KT. DNA methylation arrays as surrogate measures of cell mixture distribution. BMC Bioinforma. 2012;13(1):86.

24. Lin L. A note on concordance correlation coefficient. Biometrics. 2000;56: 324-5.

25. Moayyeri A, Hammond CJ, Valdes AM, Spector TD. Cohort profile: TwinsUK and healthy ageing twin study. Int J Epidemiol. 2013;42(1):76-85.

26. Mangino M, Hwang SJ, Spector TD, Hunt SC, Kimura M, Fitzpatrick AL, Christiansen L, Petersen I, Elbers CC, Harris T, et al. Genome-wide meta-analysis points to CTC1 and ZNF676 as genes regulating telomere homeostasis in humans. Hum Mol Genet. 2012;21(24):5385-394.

27. Grundberg E, Small KS, Hedman ÅK, Nica AC, Buil A, Keildson S, Bell JT, Yang TP, Meduri E, Barrett A, et al. Mapping cis-and trans-regulatory effects across multiple tissues in twins. Nat Genet. 2012:44(10):1084-89.

28. Speed D, Balding DJ. Relatedness in the post-genomic era: is it still useful? Nat Rev Genet. 2015;16(1):33-44.

29. Frentiu FD, Clegg SM, Chittock J, Burke T, Blows MW, Owens IP. Pedigree-free animal models: the relatedness matrix reloaded. Proc $\mathrm{R}$ Soc Lond B Biol Sci. 2008;275(1635):639-47.

\section{Submit your next manuscript to BioMed Central and we will help you at every step:}

- We accept pre-submission inquiries

- Our selector tool helps you to find the most relevant journal

- We provide round the clock customer support

- Convenient online submission

- Thorough peer review

- Inclusion in PubMed and all major indexing services

- Maximum visibility for your research

Submit your manuscript at www.biomedcentral.com/submit 